\title{
Investigating various performance traits of Karakul sheep
}

\author{
Halil EROL ${ }^{1, a, \varpi}$, Ceyhan ÖZBEYAZ ${ }^{2, b}$, Necmettin ÜNAL ${ }^{2, c}$ \\ ${ }^{1}$ Edremit Directorate of District Agriculture and Forestry, Balıkesir; ${ }^{2}$ Ankara University, Faculty of Veterinary Medicine, Department \\ of Animal Breeding and Husbandry, Ankara, Turkey. \\ aORCID: 0000-0001-9728-7244; bORCID: 0000-0002-3748-9992; ' $O R C I D: 0000-0001-5250-7063$.
}

Corresponding author: erol.halil@ tarimorman.gov.tr

Received date: 04.12.2018- Accepted date: 20.09 .2019

\begin{abstract}
Karakul sheep in Turkey is an endangered breed according to the total number of ewes. Two herds of Karakul sheep in Tokat Province, reared similar environmental conditions, kept in situ in vivo conservation as a gene resource. This study aimed to determine various performance traits of Karakul sheep reared in breeder conditions as a gene resource. Least squares means for lambing rate (LR), litter size (LS) and lamb production (LP) were found $95.8 \pm 0.60 \%, 1.04 \pm 0.01$ and $100.0 \pm 0.80 \%$, respectively. It was determined that farm and age had significant effect on LR and LP, while farm and year had significant impact on LS. Survival rate in lambs were found 95.2 and $94.1 \%$, respectively on $90^{\text {th }}$ and $180^{\text {th }}$ days. Means of least squares for live weights of lambs at birth and on $90^{\text {th }}$ and $180^{\text {th }}$ days were identified as $3.35 \pm 0.02,21.52 \pm 0.27$ and $30.34 \pm 0.32 \mathrm{~kg}$, respectively. Live weight after shearing and greasy fleece weight values were $40.73 \pm 0.12$ and $2.04 \pm 0.01 \mathrm{~kg}$ for females and $62.65 \pm 0.53$ and $3.48 \pm 0.06 \mathrm{~kg}$ for males. Lactation milk yield (LMY) was $104.85 \pm 3.73 \mathrm{~kg}$ in ewes and lactation duration was $159.01 \pm 1.70$ days. LMY was affected by the lactation number and farm while lactation duration was affected by lactation number. Results revealed that various performance traits of Karakul sheep conserved in breeder conditions were similar to or better than those previously reported for this breed. Also, the breed is similar to medium size native sheep breeds in terms of growth and mature live weight.
\end{abstract}

Keywords: Genetic resource, Karakul sheep, production traits

\section{Karagül koyunlarında çeşitli verim özelliklerinin araştırılması}

Özet: Türkiye'de Karagül koyunu, yok olma tehlikesi altında bulunan bir ırktır. Tokat ilinde birbirine yakın ve benzer çevresel koşullarda yetiştirilen iki Karagül koyun sürüsü, yetiştirici koşullarında gen kaynağı olarak korunmaktadır. Bu araştırma, yetiştirici koşullarında gen kaynağı olarak korunan Karagül koyunlarında çeşitli verim özelliklerinin incelenmesi amacıyla yapılmıştır. Koyunlarda doğum oran1, bir doğuma kuzu sayısı ve kuzu verimi için en küçük kareler ortalamaları \% 95,8 $\pm 0,60 ; 1,04 \pm 0,01$ ve \% $100,0 \pm 0,80$ olmuştur. İşletme ve yaşın doğum oranına ve kuzu verimine; işletme ve yılın ise bir doğuma kuzu sayısına etkileri önemli bulunmuştur. Kuzularda 90. ve 180. günde yaşama gücü sirasiyla \% 95,2 ve 94,1 olarak tespit edilmiştir. Kuzularda doğum, 90 . ve

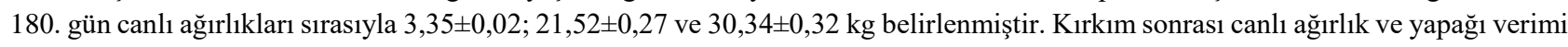
dişilerde $40,73 \pm 0,12$ ve $2,04 \pm 0,01 \mathrm{~kg}$, erkeklerde $62,65 \pm 0,53$ ve $3,48 \pm 0,06 \mathrm{~kg}$ tespit edilmiştir. Koyunlarda laktasyon süt verimi

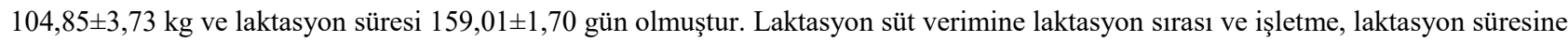
ise laktasyon sırasının etkisi önemli olmuştur. Sonuç olarak yetiştirici koşullarında korunan Karagül koyunlarında genel olarak çeşitli verim özelliklerinin ırk için bildirilen değerlere benzer veya daha iyi olduğu belirlenmiştir. Ayrıca Karagül ırkı, canlı ağırlık ve büyüme bakımından orta yapılı yerli koyun irklarına benzerlik göstermektedir.

Anahtar sözcükler: Gen kaynă̆ı, Karagül koyunu, verim özellikleri

\section{Introduction}

Karakul is a fat-tailed sheep breed with coarse fleece (1). This breed is called after Karakul town in Turkmenistan (1) or Karagöl located in the city of Bukhara in Uzbekistan (2). The most significant characteristic of this breed is the Astrakhan fur obtained from the newborn lambs. It has been reported that Karakul breed was first brought to Tokat and Antalya in Turkey by the families who migrated from Caucasia at the end of $19^{\text {th }}$ century
(22). Later, rams and ewes were brought from Turkistan in 1929 to start breeding $(2,11)$. Breeding was undertaken in many state institutions led by Çifteler (Eskişehir) and Kazova (Tokat); however, subsequently breeding in these state farms was terminated. Today, Karakul sheep are bred only in Tokat vicinity at a small scale. Because total number of Karakul ewes has decreased considerably, Karakul breed in Turkey was accepted as an endangered breed (8). Therefore, two herds (a total of 320-head) were 
conserved in the framework of the project to conserve the genetic resources of domesticated animals. Previous studies, carried out in the 1960s, investigating Karakul sheep in Turkey were mostly related to curl forms and skin structure $(6,7,11)$. Some studies performed in 2000s were published on milk (16), fleece (17), fertility (23), and some production traits (13). A number of production traits of ewes and lambs in the Karakul herds during the period of 2005-2008, in which the current research was conducted, were reported (13). The current study includes fertility, live weight after shearing, greasy fleece yield, milk yield and some udder measurements along with lamb survival rates and growth characteristics of the herds from 2011 to 2015.

Karakul sheep breed has been reared for a long time in Anatolia and it is one of components of biodiversity of Turkey. This breed faces a challenge of the need to increase production traits to provide sustainable production. Astrakhan fur, the most important yield of the breed, is not generally utilized in Turkey, and so the existence of the breed depends on the use of other yield characteristics. For this reason, it is important to know the current information about production traits of the breed.

The study aimed to investigate fertility, survival ability, growth, production of milk and wool of Karakul sheep under in situ in vivo conservation.

\section{Material and Methods}

The study was conducted on two Karakul sheep herds reared in Gülpınar and Ulaş villages of Tokat province (Gülpınar and Ulaş villages are situated between $40^{\circ} 18^{\prime} 03^{\prime \prime}-40^{\circ} 18^{\prime} 48^{\prime \prime}$ east longitude and $36^{\circ} 26^{\prime} 11^{\prime \prime}-36^{\circ}$ 23' $07^{\prime \prime}$ north latitude and elevation from sea level is 630 $\mathrm{m})$. Data on the number of ewes, the performance traits of which were under investigation are presented in the form of tables. Husbandry and feeding conditions were generally similar in the farms where the project was carried out. The distance between the two farms is $7 \mathrm{~km}$. Ewes in both herds were fed on pasture during the first $3 / 5$ period of gestation, and were offered with $400 \mathrm{~g}$ of concentrate feed $(14.0 \%$ crude protein and $2400 \mathrm{kcal}$ $\mathrm{ME} / \mathrm{kg}$ ) and roughage (400 g sugar beet pulp and $500 \mathrm{~g}$ vetch plus barley straw) per ewe daily during the last $2 / 5$ period of gestation. The ewes were kept indoors during the first 6 weeks of lactation period and fed similar to the last period of gestation. After indoor keeping, the ewes were fed on pasture during the rest of lactation period.

Ewes mating was conducted as random mating. Lambing rate (LR) and lamb production (LP) were calculated according to the number of ewes exposed to rams and litter size (LS) was calculated based on the number of ewes lambing. Survival rate (SR) of lambs was based on lambs born alive. The date of birth, gender and age of dam were recorded at birth along with their birth weight no later than 24 hours after the birth. The lambs were received alfalfa hay and lamb grower feed. The growth of lambs was recorded and live weight on the $90^{\text {th }}$ and $180^{\text {th }}$ days was calculated by using interpolation method. Ewes were shorn in June every year. Greasy fleece weight and live weight after shearing were identified with the help of electronic bascules sensitive to $50 \mathrm{~g}$.

Milk yield controls were conducted in 2013 on a total of 30 ewes per herd randomly selected from both herds with single births at the second week of February and were in the $1^{\text {st }}, 2^{\text {nd }}$ and $3^{\text {rd }}$ lactation. The first milk control was conducted about on the $45^{\text {th }}$ day after the birth and controls were continued about 90 and 135 days after parturition. The data for the lactation milk yield (LMY) were calculated by interpolation and extrapolation methods. The lactation duration (LD) was calculated as the period between the date of birth and the end of lactation. The end of lactation was determined by extrapolation method based on the last milk control day.

The lambs were separated from their mother one day before the milk control day at $17^{.00}$ and the ewes were milked by hand on the milk control day around at $08 .{ }^{00}$ and 17. ${ }^{00}$. Lambs in both herds were not weaned until the last control milking and went to the pasture with the ewes. LMY was calculated by using Fleischmann's method (TrapezII). Udder measurements were collected right before the $1^{\text {st }}$ and the $3^{\text {rd }}$ milk control days of lactation with the help of measuring tape and digital calipers (18).

Statistical analysis: Fertility, greasy fleece weight, lactation milk yield, lactation duration and udder measurements in ewes and growth characteristics in lambs were examined with the Least Squares Method. Duncan's Multiple Comparison Test was used to compare more than two groups with significant differences. Survival rate in lambs was analyzed using Chi-Square Method (9).

\section{Results}

Table 1 presents the ewes fertility characteristics of which were assessed and Table 2 presents the least squares means for fertility characteristics. Mean least squares for LR, LS and LP in ewes were $95.8 \pm 0.60 \%, 1.04 \pm 0.01$ and $100.0 \pm 0.80 \%$, respectively. It was found that farm and age $(\mathrm{P}<0.05)$ affected LR, farm $(\mathrm{P}<0.001)$ and year $(\mathrm{P}<0.01)$ affected LS and farm $(\mathrm{P}<0.001)$ and age $(\mathrm{P}<0.05)$ affected LP (Table 2).

Survival rates of lambs on the $90^{\text {th }}$ and $180^{\text {th }}$ days were found 95.2 and $94.1 \%$ (Table 3) and the impact of the examined factors were generally insignificant. Table 4 presents the means of the live weights of the lambs at different stages. Live weights at birth and the $90^{\text {th }}$ and $180^{\text {th }}$ days of lambs were found $3.35 \pm 0.02,21.52 \pm 0.27$ and $30.34 \pm 0.32 \mathrm{~kg}$, respectively. The effects of the examined factors on the live weight of lambs at different periods were found significant at different levels $(\mathrm{P}<0.05$; $\mathrm{P}<0.01 ; \mathrm{P}<0.001)$. 
Table 1. Number of ewes investigated for the fertility traits by the farm and year subclasses

\begin{tabular}{|c|c|c|c|c|c|c|}
\hline Farm & Age & 2011-2012 & 2012-2013 & 2013-2014 & 2014-2015 & General \\
\hline \multirow{6}{*}{ Gülpınar } & 2 & 38 & 26 & 16 & 35 & 115 \\
\hline & 3 & 46 & 34 & 27 & 16 & 123 \\
\hline & 4 & 24 & 47 & 33 & 27 & 131 \\
\hline & 5 & 22 & 21 & 43 & 32 & 118 \\
\hline & $6 \geq$ & 20 & 29 & 38 & 67 & 154 \\
\hline & Total & 150 & 157 & 157 & 177 & 641 \\
\hline \multirow{6}{*}{ Ulaş } & 2 & 35 & 18 & 27 & 25 & 105 \\
\hline & 3 & 35 & 36 & 18 & 27 & 116 \\
\hline & 4 & 24 & 31 & 35 & 18 & 108 \\
\hline & 5 & 22 & 23 & 30 & 35 & 110 \\
\hline & $6 \geq$ & 24 & 32 & 39 & 53 & 148 \\
\hline & Total & 140 & 140 & 149 & 158 & 587 \\
\hline \multirow{6}{*}{ General } & 2 & 73 & 44 & 43 & 60 & 220 \\
\hline & 3 & 81 & 70 & 45 & 43 & 239 \\
\hline & 4 & 48 & 78 & 68 & 45 & 239 \\
\hline & 5 & 44 & 44 & 73 & 67 & 228 \\
\hline & $6 \geq$ & 44 & 61 & 77 & 120 & 302 \\
\hline & Total & 290 & 297 & 306 & 335 & 1228 \\
\hline
\end{tabular}

Table 2. Numerical values and the least squares means ( \pm SEM) for fertility traits

\begin{tabular}{|c|c|c|c|c|c|c|c|c|}
\hline \multirow{2}{*}{ Items } & \multirow{2}{*}{ NME } & \multirow{2}{*}{ NLE } & \multirow{2}{*}{ NSLE } & \multirow{2}{*}{ NMLE } & \multirow{2}{*}{ NLBA } & \multicolumn{3}{|c|}{$\mathbf{L S M} \pm \mathbf{S E}$} \\
\hline & & & & & & LR (\%) & LP $(\%)$ & LS (x100) \\
\hline Farm & & & & & & $*$ & $* * *$ & $* * *$ \\
\hline Gülpınar & 641 & 606 & 592 & 14 & 620 & $94.4 \pm 0.80$ & $96.7 \pm 1.10$ & $102.4 \pm 0.80$ \\
\hline Ulaş & 587 & 571 & 535 & 36 & 607 & $97.1 \pm 0.80$ & $103.4 \pm 1.20$ & $106.5 \pm 0.80$ \\
\hline Age & & & & & & $*$ & $*$ & - \\
\hline 2 & 220 & 202 & 197 & 5 & 207 & $91.9 \pm 1.30^{\mathrm{a}}$ & $94.4 \pm 1.90^{\mathrm{a}}$ & $102.6 \pm 1.40$ \\
\hline 3 & 239 & 232 & 224 & 8 & 240 & $97.3 \pm 1.30^{\mathrm{b}}$ & $100.3 \pm 1.80^{\mathrm{b}}$ & $103.1 \pm 1.30$ \\
\hline 4 & 239 & 228 & 215 & 13 & 241 & $95.5 \pm 1.30^{\mathrm{b}}$ & $100.9 \pm 1.80^{\mathrm{b}}$ & $105.6 \pm 1.30$ \\
\hline 5 & 228 & 221 & 206 & 15 & 236 & $96.8 \pm 1.30^{\mathrm{b}}$ & $103.9 \pm 1.90^{\mathrm{b}}$ & $107.3 \pm 1.30$ \\
\hline $6 \geq$ & 302 & 294 & 285 & 9 & 303 & $97.2 \pm 1.20^{\mathrm{b}}$ & $100.6 \pm 1.70^{\mathrm{b}}$ & $103.6 \pm 1.20$ \\
\hline Year & & & & & & - & - & $* *$ \\
\hline 2011-2012 & 290 & 275 & 264 & 11 & 286 & $95.1 \pm 1.20$ & $99.2 \pm 1.70$ & $104.3 \pm 1.20^{\mathrm{ab}}$ \\
\hline $2012-2013$ & 297 & 283 & 260 & 23 & 306 & $95.2 \pm 1.20$ & $103.1 \pm 1.70$ & $108.3 \pm 1.20^{\mathrm{b}}$ \\
\hline 2013-2014 & 306 & 296 & 288 & 8 & 304 & $96.5 \pm 1.10$ & $98.9 \pm 1.60$ & $102.4 \pm 1.20^{\mathrm{a}}$ \\
\hline 2014-2015 & 335 & 323 & 315 & 8 & 331 & $96.3 \pm 1.10$ & $98.9 \pm 1.60$ & $102.6 \pm 1.10^{\mathrm{a}}$ \\
\hline \multicolumn{9}{|l|}{ Interactions } \\
\hline FxA & & & & & & - & - & - \\
\hline FxY & & & & & & - & - & - \\
\hline AxY & & & & & & - & - & - \\
\hline General & 1228 & 1177 & 1127 & 50 & 1227 & $95.8 \pm 0.60$ & $100.0 \pm 0.80$ & $104.4 \pm 0.60$ \\
\hline
\end{tabular}

F: Farm, A: Age, Y: Year, - $\mathrm{P}>0.05, * \mathrm{P}<0.05, * * \mathrm{P}<0.01, * * * \mathrm{P}<0.001$

${ }_{\mathrm{a}, \mathrm{b}}$ Differences between the means with unlike letters in the same column are significant at $\mathrm{P}<0.05$.

LSM: Least squares means, SE: Standard error, NME: Number of mating ewes, NLE: Number of lambing ewes, NSLE: Number of single lambing ewes, NMLE: Number of multiple lambing ewes, NLBA: Number of lambs born alive, LR: Lambing rate, LP: Lamb production, LS: Litter size 
Table 3. Number of lambs born alive and survival rates at different periods

\begin{tabular}{|c|c|c|c|c|c|}
\hline \multirow[t]{2}{*}{ Items } & \multicolumn{3}{|c|}{ Number of lambs (n) } & \multicolumn{2}{|c|}{ Survival rate $(\%)$} \\
\hline & Live birth & $90^{\text {th }}$ day & $180^{\text {th }}$ day & $9^{\text {th }}$ day & $180^{\text {th }}$ day \\
\hline Farm & & & & $*$ & $*$ \\
\hline Gülpınar & 620 & 602 & 596 & 96.9 & 96.0 \\
\hline Ulaş & 607 & 567 & 560 & 93.4 & 92.3 \\
\hline Dam Age & & & & - & - \\
\hline 2 & 207 & 194 & 192 & 93.7 & 92.8 \\
\hline 3 & 240 & 230 & 227 & 95.8 & 94.2 \\
\hline 4 & 241 & 233 & 232 & 96.7 & 96.3 \\
\hline 5 & 236 & 228 & 226 & 96.2 & 95.8 \\
\hline $6 \geq$ & 303 & 284 & 279 & 93.7 & 92.1 \\
\hline Birth Year & & & & - & - \\
\hline 2012 & 286 & 271 & 268 & 94.8 & 93.4 \\
\hline 2013 & 306 & 288 & 283 & 94.1 & 92.5 \\
\hline 2014 & 304 & 294 & 293 & 96.4 & 96.4 \\
\hline 2015 & 331 & 316 & 312 & 95.5 & 94.3 \\
\hline Gender & & & & - & - \\
\hline Female & 603 & 572 & 565 & 94.7 & 93.7 \\
\hline Male & 624 & 597 & 591 & 95.7 & 94.6 \\
\hline Birth Type & & & & - & - \\
\hline Single & 1127 & 1077 & 1066 & 95.5 & 94.5 \\
\hline Twin & 100 & 92 & 90 & 92.0 & 90.0 \\
\hline General & 1227 & 1169 & 1156 & 95.2 & 94.1 \\
\hline
\end{tabular}

- $\mathrm{P}>0.05, * \mathrm{P}<0.05$

Table 4. The least squares means $( \pm$ SEM) for live weight at different ages $(\mathrm{kg})$

\begin{tabular}{|c|c|c|c|c|c|c|}
\hline Items & $\mathrm{n}$ & Birth & $\mathrm{n}$ & $90^{\text {th }}$ day & $\mathrm{n}$ & $180^{\text {th }}$ day \\
\hline Farm & & $* *$ & & $*$ & & $* *$ \\
\hline Gülpınar & 620 & $3.28 \pm 0.04$ & 602 & $22.01 \pm 0.41$ & 596 & $29.55 \pm 0.49$ \\
\hline Ulaş & 607 & $3.41 \pm 0.03$ & 567 & $21.03 \pm 0.30$ & 560 & $31.13 \pm 0.35$ \\
\hline Dam Age & & - & & - & & - \\
\hline 2 & 207 & $3.22 \pm 0.07$ & 194 & $21.55 \pm 0.71$ & 192 & $29.67 \pm 0.84$ \\
\hline 3 & 240 & $3.34 \pm 0.05$ & 230 & $21.90 \pm 0.48$ & 227 & $30.88 \pm 0.57$ \\
\hline 4 & 241 & $3.42 \pm 0.04$ & 233 & $22.15 \pm 0.44$ & 232 & $30.92 \pm 0.53$ \\
\hline 5 & 236 & $3.34 \pm 0.04$ & 228 & $21.58 \pm 0.44$ & 226 & $30.92 \pm 0.54$ \\
\hline $6 \geq$ & 303 & $3.41 \pm 0.04$ & 284 & $20.42 \pm 0.46$ & 279 & $29.32 \pm 0.57$ \\
\hline Birth Year & & $* * *$ & & $* * *$ & & $* * *$ \\
\hline 2012 & 286 & $3.18 \pm 0.04^{\mathrm{a}}$ & 271 & $19.14 \pm 0.47^{\mathrm{a}}$ & 268 & $28.08 \pm 0.57^{\mathrm{a}}$ \\
\hline 2013 & 306 & $3.41 \pm 0.03^{b}$ & 288 & $22.41 \pm 0.35^{b}$ & 283 & $30.00 \pm 0.43^{b}$ \\
\hline 2014 & 304 & $3.48 \pm 0.05^{\mathrm{b}}$ & 294 & $22.78 \pm 0.53^{b}$ & 293 & $30.88 \pm 0.63^{b}$ \\
\hline 2015 & 331 & $3.32 \pm 0.05^{\mathrm{c}}$ & 316 & $21.75 \pm 0.54^{\mathrm{c}}$ & 312 & $32.40 \pm 0.63^{\mathrm{c}}$ \\
\hline Gender & & $* * *$ & & $*$ & & *** \\
\hline Female & 603 & $3.23 \pm 0.03$ & 572 & $21.16 \pm 0.33$ & 565 & $29.53 \pm 0.39$ \\
\hline Male & 624 & $3.47 \pm 0.03$ & 597 & $21.88 \pm 0.35$ & 591 & $31.15 \pm 0.42$ \\
\hline Birth Type & & $* * *$ & & $*$ & & *** \\
\hline Single & 1127 & $3.76 \pm 0.01$ & 1077 & $22.10 \pm 0.11$ & 1066 & $31.72 \pm 0.13$ \\
\hline Twin & 100 & $2.93 \pm 0.05$ & 92 & $20.94 \pm 0.53$ & 90 & $28.96 \pm 0.63$ \\
\hline \multicolumn{7}{|l|}{ Interactions } \\
\hline FxDA & & $* * *$ & & - & & - \\
\hline FxBY & & $* * *$ & & $* * *$ & & $* * *$ \\
\hline FxG & & - & & $*$ & & $* * *$ \\
\hline FxBT & & - & & - & & - \\
\hline DAxBY & & $* *$ & & - & & - \\
\hline DAxG & & - & & - & & - \\
\hline DAxBT & & - & & - & & - \\
\hline BYxG & & $* *$ & & - & & - \\
\hline BYxBT & & $* * *$ & & - & & - \\
\hline GxBT & & - & & - & & - \\
\hline Regression & & & & $1.603 \pm 0.291 * * *$ & & $1.564 \pm 0.356^{* * *}$ \\
\hline General & 1227 & $3.35 \pm 0.02$ & & $21.52 \pm 0.27$ & & $30.34 \pm 0.32$ \\
\hline
\end{tabular}

F: Farm, DA: Dam Age, BY: Birth Year, G: Gender, BT: Birth Type, - $\mathrm{P}>0.05, * \mathrm{P}<0.05, * * * \mathrm{P}<0.001$

$a, b, c$ Differences between the means with unlike letters in the same column are significant at $\mathrm{P}<0.05$.

Regression: Partial regression of live weight on birth weight. 
Live weight after shearing was $40.73 \pm 0.12$ and $62.65 \pm 0.53$ and greasy fleece weight were $2.04 \pm 0.01$ and $3.48 \pm 0.06 \mathrm{~kg}$ in ewes and rams, respectively. The impact of the examined factors were found significant in general ( $\mathrm{P}<0.05 ; \mathrm{P}<0.01 ; \mathrm{P}<0.001)$, (Table 5).

Table 6 presents the least squares means for LMY and LD, and Table 7 shows the least squares means for udder measurements. LMY and LD were determined to be
$104.85 \pm 3.73 \mathrm{~kg}$ and $159.01 \pm 1.70$ days, respectively. Udder circumference on the $45^{\text {th }}$ day of lactation was found $44.20 \pm 0.37 \mathrm{~cm}$ and teat-floor distance $26.48 \pm 0.15$ $\mathrm{cm}$. It was identified that lactation number and farm affected LMY ( $\mathrm{P}<0.01)$ and lactation number affected the $\mathrm{LD}(\mathrm{P}<0.05)$. The udder measurement values except teatfloor distance was found to decrease when lactation progressed (Table 7).

Table 5. The least squares means $( \pm \mathrm{SEM})$ for live weight after shearing and greasy fleece weight $(\mathrm{kg})$

\begin{tabular}{|c|c|c|c|c|c|c|c|c|}
\hline \multirow[t]{2}{*}{ Items } & \multicolumn{5}{|c|}{ Live weight after shearing } & \multicolumn{3}{|c|}{ Greasy fleece weight } \\
\hline & $\mathbf{n}$ & Ewes & $\mathbf{n}$ & Rams & $\mathbf{n}$ & Ewes & $\mathbf{n}$ & Rams \\
\hline Farm & & $* * *$ & & $* * *$ & & $* * *$ & & $*$ \\
\hline Gülpınar & 692 & $40.23 \pm 0.13$ & 49 & $60.78 \pm 0.73$ & 685 & $1.88 \pm 0.02$ & 49 & $3.43 \pm 0.09$ \\
\hline Ulaş & 628 & $41.23 \pm 0.14$ & 60 & $64.52 \pm 0.79$ & 628 & $2.20 \pm 0.02$ & 60 & $3.54 \pm 0.08$ \\
\hline Age & & $* * *$ & & $* * *$ & & $* * *$ & & $* * *$ \\
\hline 1.5 & 164 & $35.54 \pm 0.28^{\mathrm{a}}$ & 48 & $42.59 \pm 0.95^{\mathrm{a}}$ & 164 & $1.91 \pm 0.03^{\mathrm{a}}$ & 48 & $2.86 \pm 0.10^{\mathrm{a}}$ \\
\hline 2.5 & 206 & $40.50 \pm 0.23^{b}$ & 26 & $63.29 \pm 0.99^{b}$ & 206 & $2.03 \pm 0.03^{\mathrm{b}}$ & 26 & $3.46 \pm 0.11^{\mathrm{b}}$ \\
\hline 3.5 & 229 & $42.60 \pm 0.22^{\mathrm{c}}$ & 18 & $69.72 \pm 1.15^{\mathrm{c}}$ & 228 & $2.12 \pm 0.03^{\mathrm{bc}}$ & 18 & $3.97 \pm 0.12^{\mathrm{c}}$ \\
\hline 4.5 & 233 & $42.43 \pm 0.22^{\mathrm{c}}$ & 17 & $74.99 \pm 1.23^{\mathrm{d}}$ & 230 & $2.14 \pm 0.03^{\mathrm{c}}$ & 17 & $3.65 \pm 0.13^{\mathrm{b}}$ \\
\hline 5.5 & 248 & $42.01 \pm 0.22^{\mathrm{c}}$ & & & 248 & $2.03 \pm 0.02^{\mathrm{b}}$ & & \\
\hline $6 \geq$ & 240 & $41.29 \pm 0.24^{b c}$ & & & 237 & $2.00 \pm 0.03^{\mathrm{b}}$ & & \\
\hline Year & & $* * *$ & & $* * *$ & & $* * *$ & & $* * *$ \\
\hline 2012 & 329 & $39.59 \pm 0.19^{\mathrm{a}}$ & 18 & $57.96 \pm 1.15^{\mathrm{a}}$ & 329 & $1.99 \pm 0.02^{\mathrm{a}}$ & 18 & $3.23 \pm 0.12^{\mathrm{a}}$ \\
\hline 2013 & 328 & $40.82 \pm 0.18^{b}$ & 29 & $62.31 \pm 1.09^{b}$ & 327 & $2.05 \pm 0.02^{\mathrm{ab}}$ & 29 & $3.24 \pm 0.12^{\mathrm{a}}$ \\
\hline 2014 & 331 & $40.44 \pm 0.20^{\mathrm{b}}$ & 31 & $63.52 \pm 1.12^{\mathrm{b}}$ & 327 & $1.97 \pm 0.02^{\mathrm{a}}$ & 31 & $3.70 \pm 0.12^{\mathrm{b}}$ \\
\hline 2015 & 332 & $42.07 \pm 0.20^{\mathrm{c}}$ & 31 & $66.81 \pm 0.92^{\mathrm{c}}$ & 330 & $2.15 \pm 0.03^{\mathrm{b}}$ & 31 & $3.76 \pm 0.10^{\mathrm{b}}$ \\
\hline \multicolumn{9}{|c|}{ Interactions } \\
\hline FxA & & - & & $* * *$ & & $* * *$ & & $* * *$ \\
\hline FxY & & $* * *$ & & $* * *$ & & $* * *$ & & - \\
\hline$A x Y$ & & $* *$ & & - & & $* * *$ & & - \\
\hline General & 1320 & $40.73 \pm 0.12$ & 109 & $62.65 \pm 0.53$ & 1313 & $2.04 \pm 0.01$ & 109 & $3.48 \pm 0.06$ \\
\hline
\end{tabular}

F: Farm, A: Age, Y: Year, - $\mathrm{P}>0.05,{ }^{*} \mathrm{P}<0.05, * * \mathrm{P}<0.01, * * * \mathrm{P}<0.001$

$\mathrm{a}, \mathrm{b}, \mathrm{c}, \mathrm{d}$ Differences between the means with unlike letters in the same column are significant at $\mathrm{P}<0.05$.

Table 6. Some descriptive values and the least squares means $( \pm$ SEM) for lactation milk yield and lactation duration

\begin{tabular}{|c|c|c|c|c|c|c|c|}
\hline Items & $\mathbf{n}$ & LMY (kg) & Min & Max & LD (day) & Min & $\operatorname{Max}$ \\
\hline $\mathrm{LN}$ & & $* *$ & & & $*$ & & \\
\hline 1 & 20 & $92.12 \pm 4.56^{\mathrm{a}}$ & 44 & 128 & $153.41 \pm 2.91^{\mathrm{a}}$ & 130 & 169 \\
\hline 2 & 19 & $112.09 \pm 4.68^{\mathrm{b}}$ & 74 & 174 & $158.24 \pm 2.99^{\mathrm{ab}}$ & 136 & 187 \\
\hline 3 & 20 & $110.33 \pm 4.56^{\mathrm{b}}$ & 85 & 141 & $165.38 \pm 2.91^{\mathrm{b}}$ & 143 & 202 \\
\hline Farm & & $* *$ & & & - & & \\
\hline Gülpınar & 29 & $97.50 \pm 3.79$ & 44 & 128 & $156.21 \pm 2.42$ & 130 & 202 \\
\hline Ulaş & 39 & $112.19 \pm 3.73$ & 74 & 174 & $161.81 \pm 2.38$ & 136 & 187 \\
\hline \multicolumn{8}{|c|}{ Interaction } \\
\hline $\mathrm{LNxF}$ & & - & & & - & & \\
\hline General & 59 & $104.85 \pm 3.73$ & 44 & 174 & $159.01 \pm 1.70$ & 130 & 202 \\
\hline
\end{tabular}

LMY: Lactation milk yield, LD: Lactation duration, LN: Lactation number, F: Farm, Min: Minimum, Max: Maximum, - P>0.05, * $\mathrm{P}<0.05, * * \mathrm{P}<0.01$

a, b Differences between the means with unlike letters in the same column are significant at $\mathrm{P}<0.05$. 
Table 7. The least squares means $( \pm \mathrm{SEM})$ for some udder measurements $(\mathrm{cm})$

\begin{tabular}{|c|c|c|c|c|c|c|c|}
\hline \multirow{2}{*}{ Items } & \multirow{2}{*}{$\mathbf{n}$} & \multicolumn{6}{|c|}{ Days } \\
\hline & & $45^{\text {th }}$ day & $135^{\text {th }}$ day & $45^{\text {th }}$ day & $135^{\text {th }}$ day & $45^{\text {th }}$ day & $135^{\text {th }}$ day \\
\hline & & \multicolumn{2}{|c|}{ Udder width } & \multicolumn{2}{|c|}{ Udder depth } & \multicolumn{2}{|c|}{ Udder circumference } \\
\hline $\mathrm{LN}$ & & $*$ & $* *$ & - & - & - & - \\
\hline 2 & 22 & $13.17 \pm 0.21^{\mathrm{a}}$ & $9.48 \pm 0.28^{\mathrm{a}}$ & $14.70 \pm 0.18$ & $11.64 \pm 0.56$ & $42.98 \pm 0.63$ & $28.73 \pm 0.88$ \\
\hline 3 & 20 & $13.95 \pm 0.22^{b}$ & $10.09 \pm 0.30^{\mathrm{ab}}$ & $15.25 \pm 0.18$ & $11.35 \pm 0.60$ & $44.78 \pm 0.65$ & $30.34 \pm 0.95$ \\
\hline 4 & 22 & $13.92 \pm 0.21^{\mathrm{b}}$ & $10.74 \pm 0.27^{b}$ & $14.94 \pm 0.18$ & $12.37 \pm 0.56$ & $44.84 \pm 0.62$ & $31.14 \pm 0.88$ \\
\hline Farm & & $* *$ & - & - & - & $* * *$ & - \\
\hline Gülpınar & 31 & $13.31 \pm 0.18$ & $9.99 \pm 0.24$ & $14.86 \pm 0.15$ & $11.16 \pm 048$ & $45.74 \pm 0.53$ & $30.50 \pm 0.75$ \\
\hline Ulaş & 33 & $14.05 \pm 0.17$ & $10.22 \pm 0.22$ & $15.06 \pm 0.14$ & $12.41 \pm 0.45$ & $42.66 \pm 0.51$ & $29.64 \pm 0.72$ \\
\hline \multicolumn{8}{|l|}{ Interaction } \\
\hline $\mathrm{LNxF}$ & & $* *$ & - & - & - & $*$ & - \\
\hline \multirow[t]{2}{*}{ General } & 64 & $13.68 \pm 0.12$ & $10.10 \pm 0.16$ & $14.96 \pm 0.10$ & $11.79 \pm 0.33$ & $44.20 \pm 0.37$ & $30.07 \pm 0.52$ \\
\hline & & \multicolumn{2}{|c|}{ Length of right teat } & \multicolumn{2}{|c|}{ Length of left teat } & \multicolumn{2}{|c|}{ Diameter of right teat } \\
\hline $\mathrm{LN}$ & & $* *$ & $*$ & $*$ & - & $*$ & $*$ \\
\hline 2 & & $2.93 \pm 0.12^{\mathrm{a}}$ & $2.25 \pm 0.07^{\mathrm{a}}$ & $3.00 \pm 0.13^{\mathrm{a}}$ & $2.34 \pm 0.07$ & $1.64 \pm 0.05^{\mathrm{a}}$ & $1.31 \pm 0.03^{\mathrm{a}}$ \\
\hline 3 & & $3.41 \pm 0.13^{b}$ & $2.46 \pm 0.07^{\mathrm{ab}}$ & $3.38 \pm 0.13^{\mathrm{ab}}$ & $2.48 \pm 0.07$ & $1.87 \pm 0.05^{\mathrm{b}}$ & $1.43 \pm 0.04^{\mathrm{b}}$ \\
\hline 4 & & $3.53 \pm 0.12^{\mathrm{b}}$ & $2.54 \pm 0.07^{\mathrm{b}}$ & $3.47 \pm 0.12^{\mathrm{b}}$ & $2.50 \pm 0.06$ & $1.80 \pm 0.05^{\mathrm{b}}$ & $1.42 \pm 0.03^{\mathrm{b}}$ \\
\hline Farm & & - & - & - & - & $* * *$ & - \\
\hline Gülpınar & & $3.27 \pm 0.10$ & $2.36 \pm 0.06$ & $3.24 \pm 0.11$ & $2.41 \pm 0.06$ & $1.88 \pm 0.04$ & $1.40 \pm 0.03$ \\
\hline Ulaş & & $3.31 \pm 0.10$ & $2.47 \pm 0.06$ & $3.32 \pm 0.10$ & $2.47 \pm 0.05$ & $1.63 \pm 0.04$ & $1.38 \pm 0.03$ \\
\hline \multicolumn{8}{|l|}{ Interaction } \\
\hline $\mathrm{LNxF}$ & & - & $*$ & - & - & - & $* *$ \\
\hline \multirow[t]{2}{*}{ General } & & $3.29 \pm 0.07$ & $2.42 \pm 0.04$ & $3.28 \pm 0.073$ & $2.44 \pm 0.04$ & $1.76 \pm 0.03$ & $1.39 \pm 0.02$ \\
\hline & & \multicolumn{2}{|c|}{ Diameter of left teats } & \multicolumn{2}{|c|}{ Distance between teats } & \multicolumn{2}{|c|}{ Teat-floor distance } \\
\hline $\mathrm{LN}$ & & - & $* *$ & - & - & - & - \\
\hline 2 & & $1.61 \pm 0.05$ & $1.27 \pm 0.03^{\mathrm{a}}$ & $16.83 \pm 0.38$ & $13.22 \pm 0.23$ & $26.64 \pm 0.26$ & $29.94 \pm 0.46$ \\
\hline 3 & & $1.71 \pm 0.05$ & $1.41 \pm 0.03^{\mathrm{b}}$ & $17.71 \pm 0.40$ & $13.29 \pm 0.25$ & $26.45 \pm 0.27$ & $29.66 \pm 0.49$ \\
\hline 4 & & $1.72 \pm 0.05$ & $1.42 \pm 0.03^{\mathrm{b}}$ & $17.75 \pm 0.38$ & $13.76 \pm 0.23$ & $26.34 \pm 0.26$ & $28.77 \pm 0.45$ \\
\hline Farm & & $* * *$ & - & - & - & - & $*$ \\
\hline Gülpınar & & $1.80 \pm 0.04$ & $1.40 \pm 0.03$ & $17.29 \pm 0.32$ & $13.27 \pm 0.20$ & $26.53 \pm 0.22$ & $30.04 \pm 0.39$ \\
\hline Ulaş & & $1.56 \pm 0.04$ & $1.34 \pm 0.02$ & $17.56 \pm 0.31$ & $13.58 \pm 0.19$ & $26.43 \pm 0.21$ & $28.87 \pm 0.37$ \\
\hline \multicolumn{8}{|c|}{ Interaction } \\
\hline $\mathrm{LNxF}$ & & - & $*$ & - & - & - & - \\
\hline General & & $1.68 \pm 0.03$ & $1.37 \pm 0.02$ & $17.43 \pm 0.22$ & $13.42 \pm 0.14$ & $26.48 \pm 0.15$ & $29.46 \pm 0.27$ \\
\hline
\end{tabular}

LN: Lactation number, F: Farm, - $\mathrm{P}>0.05, * \mathrm{P}<0.05, * * \mathrm{P}<0.01, * * * \mathrm{P}<0.001$

a, b Differences between the means with unlike letters in the same column are significant at $\mathrm{P}<0.05$.

\section{Discussion and Conclusion}

Lambing rate (LR) (95.8\%) obtained in this study is a rather high value for native sheep breeds. As a matter of fact, LR values were found higher than the values reported for the same breed $(85.0-91.4 \%)(13,15,23)$ and some of the other fat tailed breeds (Akkaraman, Morkaraman) $(67.6-90.5 \%)(3,12)$. The high lambing rate in both farms indicates that environmental conditions were taken into consideration during the mating period. Fertility characteristics were considered to be the lowest in two years old ewes. Litter size (LS) obtained from Karakul ewes (1.04) was identified similar to the values reported for the same breed $(1.00-1.18)(11,13,15,23)$. The
Karakul breed has of low value in terms of LS and is similar to the Dağlıç (1.05) (10) and Karayaka (1.03 $1.08)(5,20)$ in this respect. The low LS in Karakul herds is due to the low rate of twin birth; this shows that although the breed has a high LR, it is not a prolific breed.

There is only one study in the literature on the survival rate (SR) of Karakul lambs in Turkey (13). In the present study, SR values at $90^{\text {th }}$ and $180^{\text {th }}$ days (95.2 and $94.1 \%$ ) were found similar to those identified for the same herds from 2006 to 2008, reported as 96.0 and $90.0 \%$, in general (13). SR values obtained from lambs on the $90^{\text {th }}$ and $180^{\text {th }}$ days can be regarded as optimal and this is important for the sustainability of the herds. 
The findings of the birth weight for female lambs $(3.23 \mathrm{~kg})$ and male lambs $(3.47 \mathrm{~kg})$ and the average live weight on the $180^{\text {th }}$ days $(30.34 \mathrm{~kg})$ in this study were similar (3.24 and $3.47 \mathrm{~kg}$ ) (11) or higher (3.03 and 3.23 $\mathrm{kg}$ ) (13) than the those of values for birth weight of female and male lambs and live weight on the $180^{\text {th }}$ day $(24.62$ $\mathrm{kg}$ ) (13) of the same breed. When the live weights at birth, $90^{\text {th }}$ and $180^{\text {th }}$ days of the breed are evaluated together, it could be said that Karakul breed is similar to medium size breeds (Bafra, Dağlıç, Karayaka), but lower than the large size breeds (Akkaraman, Chios) in terms of the growth (1, $4,5,12,19,21)$.

The means obtained for the ewes live weight after shearing $(40.73 \mathrm{~kg})$ and greasy fleece weight $(2.04 \mathrm{~kg})$ were consistent with the those of results reported for the same breed (36.81 - $42.95 \mathrm{~kg}$ and $1.84-2.84 \mathrm{~kg})(11,13)$. In addition, live weight after shearing value was similar to the lower limit of range $(42.70-62.60 \mathrm{~kg})$ reported for some native breeds $(5,19)$.

LMY (104.85 kg) obtained in the current study was found higher than the means reported for the same breed $(61.5$ and $60.0 \mathrm{~kg})(16,22)$. The herd in Ulaş village is regularly milked each year; however, the herd in Gülpınar village was milked for the first time in the framework of the study. The fact that Ulaş herd gave $14.69 \mathrm{~kg}$ more milk than Gülpınar herd might be due to accustomed to hand milking. LMY and LD differed from the lactation number groups, while the first lactation ewes had numerically the lowest values than those of the other groups. This is in the line with the general understanding that milk production of ewes generally increases by lactation numbers. In this research, high lactation milk yield of the ewes shows that breeders can utility from Karakul breed in terms of milk yield. LD determined in this study (159.01 days) was found in the range of values reported for the same breed (137.9 - 168.0 days) $(2,16,22)$. On the other hand, LD was similar in both herds. There was a wide variation in LMY and LD in both herds. This shows that it is possible to make improvement in the direction of milk yield in Karakul breed.

The longer the lactation duration was in Karakul sheep, the more decreasing was observed in udder measurement values other than the teat-floor distance. This is related to decreased milk yield due to progression of lactation. As a matter of fact, similar situation has been reported for various native breeds $(3,18)$. In general, udder measurement values obtained for Karakul ewes in this study were higher than those found for Tushin and Morkaraman ewes (14). Udder measurement values obtained for Bafra sheep (18) on the $42^{\text {nd }}$ day of lactation were similar to or higher than the values found in the current study other than the values for right and left teat length and the distance between teats.
In conclusion, the performance traits of Karakul sheep were similar to or better than the those of results reported before for the same breed, and the breed was alike to medium size native breeds in terms of growth and mature live weight.

\section{Acknowledgements}

The authors would like to thank General Directorate of Agricultural Research and Policies, Ministry of Agriculture and Forestry, for their contribution to the implementation of the project.

\section{Financial Support}

This research received no grant from any funding agency/sector.

\section{Conflict of Interest}

The authors declared that there is no conflict of interest.

\section{References}

1. Akçapınar H (2000): Koyun yetiştiriciliği. ISBN: 97596978-1-5, Ankara

2. Aköz K (1961): Karagül koyunu ve Karagül yetiştiriciliği. Lalahan Zootekni ve Araştırma Enstitüsü, Ankara.

3. Akçapınar H, Kadak R, Odabaşıoğlu F (1982): Morkaraman ve Kangal-Akkaraman koyunlarinin döl verimi ve süt verimi üzerinde karşılaştırmalı araştırmalar. Ankara Univ Vet Fak Derg, 3-4, 379-391.

4. Akçapınar H, Özbeyaz C, Ünal N, et al (2000): The possibilities of developing dam and sire lines using Akkaraman, Saklz and Klvirclk sheep breeds for lamb production I. Fertility in Akkaraman sheep, survival rate and growth characteristics of Sakiz $x$ Akkaraman $F_{1}$ and Kivırcı $x$ Akkaraman $F_{1}$ lambs. Turk J Vet Anim Sci, 24, 71-79.

5. Akçapınar H, Ünal N, Atasoy F, et al (2002): Adaptation capability of Karayaka and Bafra (Chios x Karayaka B $B_{1}$ ) genotypes reared in Lalahan Livestock Research conditions. Lalahan Hay Araşt Enst Derg, 1, 11-24.

6. Aköz K, Öznacar K (1960): A comparative study on the forms of curly, brightness of the curls and birth weigths of purebred Afgan Karakul lambs' raised at Lalahan Animal Breeding Station and purebred Karakul lambs' raised at Çifteler Hara. Lalahan Zoot Araş Enst Derg, 7, 65-78.

7. Aköz K (1960): The relationship between finest of the Karakul ewes wool fibers and curle conformation of the new born Karakul lambs. Lalahan Zoot Araşt Enst Derg, 7, 2127.

8. Anonymous (2015): Animal genetic resources for food and agriculture, The second report on the state of the world's. FAO Commission on Genetic Resources for Food and Agriculture Assessments, Rome.

9. Anonymous (2008): SPSS Software, Statistical Package for the Social Sciences for Windows. Statistical Innovations Inc (Version 14.01, No: 9869264), USA.

10. Başpınar H (1985): A comparative study on the fertility, milk yield and fleece yield performances of main sheep 
breeds in Turkey, kept under semi-intensive conditions. İstanbul Üniv Vet Fak Derg, 2, 43-66.

11. Batu S, Özcan H (1966): The most important morphological characters and yields of the Karakül sheep and the breeding system at Çifteler Stud Farm. Lalahan Zoot Araşt Enst Derg, 1-2, 135-139.

12. Çolakoğlu N, Özbeyaz C (1999): Comparison of some production traits in Malya and Akkaraman Sheep. Turk $\mathbf{J}$ Vet Anim Sci, 23, 351-360.

13. Erol H, Akçadağ Hİ (2009): Some production characteristics of Karagül sheep in situ conditions. Lalahan Hay Araşt Enst Derg, 46, 91-104.

14. Kırmizibayrak T, Aksoy AR, Saatci M, et al (2005): Milk yield and udder charecteristics in Tuj and Morkaraman ewes and the relationships between them. Kafkas Üniv Vet Fak Derg, 1, 11-15.

15. Köseoğlu H (1978): Studies on the improvement of twinning rate with the use of hormones in Karakul sheep. Lalahan Zoot Araşt Enst Derg, 3-4, 64-67.

16. Küçük M, Öztürk Y, Bayram D (2000): Comparison of milk yield characteristics on Hamdani, Karagül and Morkaraman breeds in semi-intensive conditions. Y.Y.Ü. Vet Fak Derg, 1, 44-48.
17. Küçük M, Yılmaz O, Ateş CY (2000): The evaluation of Morkaraman, Hamdani and Karakul wool for carpet wool type. YYÜ Vet Fak Derg, 2, 54-59.

18. Ünal N, Akçapınar H, Atasoy F, et al (2008): Some udder traits and growth of lambs and phenotypic correlations between those of traits with milking traits and milk production measured by various milk estimation methods in Bafra sheep. Ankara Univ Vet Fak Derg, 55, 117-124.

19. Ünal N, Akçapınar H, Atasoy H, et al (2004): The body weight and fleece traits of White Karaman, Chios $x$ White Karaman $F_{l}, B_{l}$, Klvirclk $x$ White Karaman $F_{l}, B_{l}$, Karayaka and Bafra sheep. Lalahan Hay Araşt Enst Derg, 2, 15-22.

20. Ünal N, Atasoy F, Akçapınar H, et al (2003): Fertility traits, survival rate and growth characteristics of Karayaka and Bafra (Chios x Karayaka $B_{1}$ ) genotypes. Turk J Vet Anim Sci, 27, 265-272.

21. Ünal N (2002): Survival rate, growth characteristics and some body measurements of Akkaraman and Sakiz (Chios) $x$ Akkaraman $F_{1}$ lambs. Turk J Vet Anim Sci, 26, 109-116.

22. Yalçın BC (1986): Sheep and goats in Turkey. FAO Animal Production and Health Papaer, Number 60, Rome.

23. Yılmaz O, Odabaşığlu F (2006): Hamdani, Morkaraman ve Karagül koyunlarında kuzulatma sıklığının artırılması olanakları. YYÜ Sağlık Bil Derg, 1, 16-126. 